\title{
Quantitative STEM for Bimetallic Catalyst Nanoparticles
}

Xiaonan Luo $^{1}$, Peter Nellist ${ }^{2}$, Sergio Lozano-Perez ${ }^{3}$, Aakash Varambhia ${ }^{4}$ and Dogan Ozkaya ${ }^{4}$

${ }^{1}$ University of Oxford, Oxford, England, United Kingdom, ${ }^{2}$ University of Oxford, Department of Materials, United States, ${ }^{3}$ University of Oxford, United States, ${ }^{4}$ Johnson Matthey, United States

Fuel cells are very promising energy-conversion devices due to their high efficiency and near-zero carbon emission footprint. Fuel cells still require a high mass of precious metal $\mathrm{Pt}$ as an electrocatalyst to ensure sufficient power output efficiency, but the sources and cost of Pt greatly limit its commercial application in fuel cells technology. Thus, the development of high-active and low-cost electrocatalysts is of much significance for the commercialization of fuel cells. Compared with Pt, bimetallic catalyst nanoparticles $(\mathrm{Pt} / \mathrm{Co})$ exhibit excellent performance with less mass of precious metal, but the intrinsic mechanism of this enhanced catalytic performance has not been fully explained. The properties of nanoparticles mainly depend on size, structure, morphology, and composition. Previous work has found that the finite size, domain structure, and composition variations can introduce strain to nanoparticles ${ }^{[1]}$. The strain has also been shown to be related to the catalytic activity of nanoparticles through density functional theory calculations $^{[2]}$. Being able to measure the local composition and to relate composition variations to the strain within nanoparticles will further our understanding of the operation of heterogeneous catalysts. In this project, we present a novel methodology to precisely measure strain distribution on atomic resolution annular dark-field (ADF) images of the bimetallic nanoparticles. Afterwards, electron energy loss spectrum (EELS) and energy dispersive X-ray (EDS) signals are recorded simultaneously to analyse the composition distribution of the Pt/Co nanoparticles.

Our proposed global strain minimization algorithm is based on the detection of atom columns on atomic resolution images in real space. A reference grid is generated and refined from a selected area of the nanoparticle. The atomic site-specific strain map is obtained by derivating the discrete displacement fields. A large ensemble of the on-axis Pt/Co nanoparticles with different sizes are imaged and strainmeasured to relate strain to the finite size and particle shape. Atomic resolution ADF images, EDS, and EELS spectrum images are acquired simultaneously. The experimental spectroscopic partial scattering cross-sections of a single atom are calibrated using needle and nanoparticle standards. To obtain the quantitative composition distribution maps of the bimetallic nanoparticles, the number of Co atoms and Pt atoms are calculated from the EELS and EDS signals, respectively. The composition variations are subsequently related to strain.

To achieve high resolution of STEM imaging, the JEOL ARM-200F electron microscope fitted with a probe-forming aberration corrector was used to acquire the ADF image of the on-axis $\mathrm{Pt} / \mathrm{Co}$ nanoparticle. Strain analysis was performed on this nanoparticle, Figure 1 shows the distribution map of lattice areal expansion ( $\mathrm{xx}+\varepsilon y \mathrm{y})$. The areal expansion map demonstrates that this bimetallic nanoparticle has strain restricted to a few outer layers. Subsequently, the spectroscopic signals are collected simultaneously on this nanoparticle. The composition distribution map obtained by quantifying these spectroscopic signals is shown in Figure 2. It indicates the Pt enrichment at the surface of this bimetallic nanoparticle.

In this work, we focus on quantifying composition variations and strain distributions on the bimetallic $\mathrm{Pt} / \mathrm{Co}$ nanoparticles to explore determinates of their enhanced catalytic activities. 


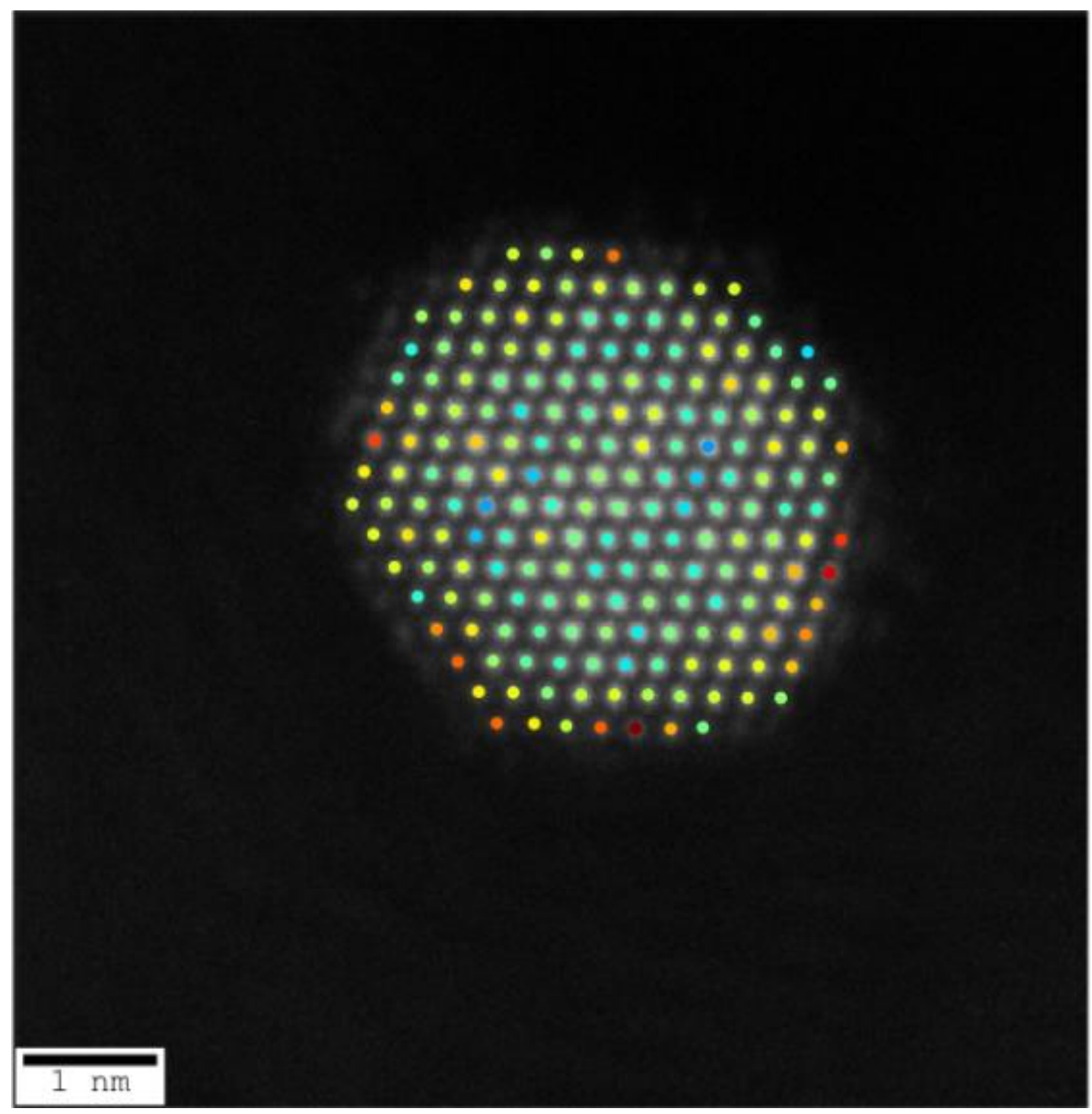

Figure 1. Figure 1. The normal strain $(\varepsilon x x+\varepsilon y y)$ distribution map of the particle. 


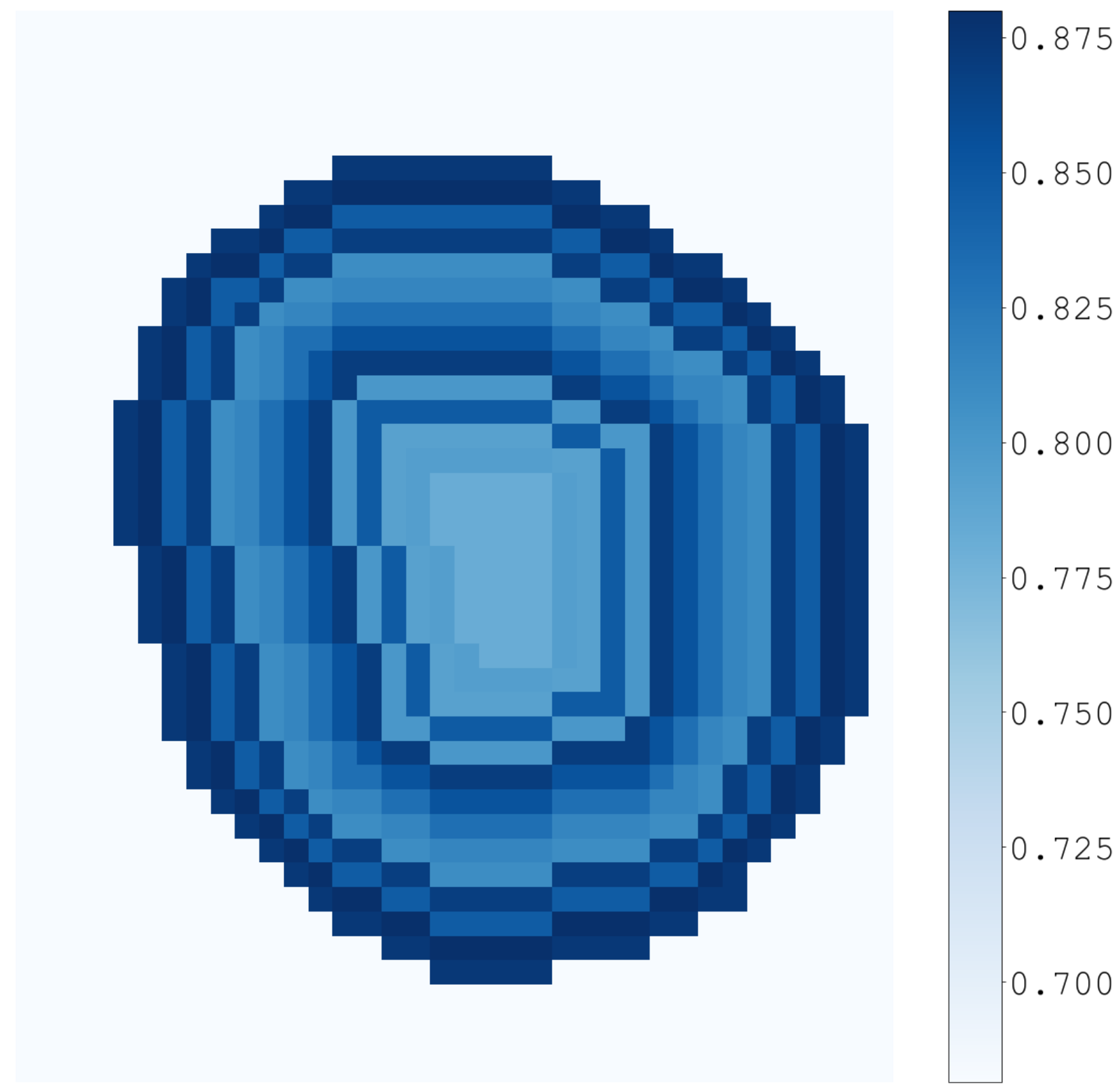

Figure 2. Figure 2. The percentage of Pt atoms in each shell from the surface to the core of the particle.

\section{References}

1. Pingel, T.N., et al., Influence of atomic site-specific strain on catalytic activity of supported nanoparticles. Nature communications, 2018. 9(1): p. 1-9.

2. Gavartin, J., et al., Exploring Fuel Cell Cathode Materials: A High Throughput Calculation Approach. ECS Transactions, 2009. 25(1): p. 1335. 